\title{
Ultrahigh temporal contrast performance of the PHELIX petawatt facility
}

\author{
V. Bagnoud ${ }^{1,2}$ and F. Wagner ${ }^{1,2}$ \\ ${ }^{1}$ Plasma physics dep., GSI Helmholtzzentrum für Schwerionenforschung GmbH, Planckstrasse 1, 64291 Darmstadt, Germany \\ ${ }^{2}$ Helmholtz Institute Jena, Fröbelstieg 3, 07743 Jena, Germany \\ (Received 17 June 2016; revised 2 September 2016; accepted 22 September 2016)
}

\begin{abstract}
We report on the temporal contrast performance of the PHELIX facility in view of the requirements imposed by solidtarget interaction experiments. The requirement analysis for the nanosecond and picosecond temporal contrast is derived from empirical data and simple theoretical modeling, while the realization shows that using an ultrafast optical parametric amplifier and plasma mirrors enables meeting this specification.
\end{abstract}

Keywords: amplified spontaneous emission; chirped-pulse amplification; optical parametric amplification; temporal contrast

\section{Introduction}

The Petawatt High-Energy Laser for heavy Ion Experiment (PHELIX) facility at GSI is a versatile dual front end glass laser system offering beam time to the international community since $2008^{[1]}$ for experiments in the fields of atomic and plasma physics, exploiting the world-wide unique capability of making combined heavy ion and laser beam experiments. The short-pulse operation mode of PHELIX is based on the chirped-pulse amplification (CPA) scheme to efficiently use the full spectral bandwidth of the glass main amplifier and produce pulses as short as $400 \mathrm{fs}$ on target. One of the drawbacks of CPA is the generation of pulses that exhibit a nanosecond pedestal related to amplified spontaneous emission (ASE) generated by the amplifier. For this reason and based on the widespread idea that the ASE is favorably reduced when the seed pulse energy is increased ${ }^{[2,3]}$, the short-pulse front end of PHELIX has been recently upgraded with a high temporal contrast amplifier module ${ }^{[4]}$. This enables the injection of energetic temporally clean short pulses in the CPA amplifier, which overcomes the limitation of standard CPA laser systems.

The generation and amplification of temporally clean short laser pulses is motivated by the necessity to avoid the generation and expansion of a pre-plasma in an unwanted and uncontrolled manner before the peak of the pulse is reached. For this reason, it is essential to understand the temporal

Correspondence to: V. Bagnoud, GSI Helmholtzzentrum für Schwerionenforschung, Planckstrasse 1, 64291 Darmstadt, Germany. Email: v.bagnoud@gsi.de contrast requirements of solid-target laser interaction from a pre-plasma generation stand point, at first. And second, a thorough analysis of the temporal degradation sources in the laser amplifier must be conducted, followed by the implementation of the solutions that exist to overcome them. Indeed, the goal is not to avoid target ionization because it is clear that the ionization of the target will be reached at some point during the intensity rise of the pulse, but rather to ensure that this has a limited effect on the interaction conditions.

In a first part, the paper revisits the theme of quantum noise in amplifiers to derive a simple description of the ASE contrast level in CPA lasers, which is put in perspective of the laser ionization threshold of materials. Then a simple plasma expansion model is used to quantify some requirements on the speed of the pulse rising front for various solid materials, where the pre-plasma is not allowed to flow excessively until the peak of the intensity is reached. In a second part, the paper describes the experimental implementation at PHELIX of two techniques aiming at controlling the ASE level by use of an ultrafast optical parametric amplifier (uOPA) and aiming at stiffening the pulse leading edge by use of one or two plasma mirrors. Altogether, this demonstrates that PHELIX operates with an ultrahigh temporal contrast level that fulfils the requirements of most of the targets commonly used in modern laser-matter interaction experiments.

\section{Requirement on the ASE level in petawatt lasers}

The ASE level in CPA lasers has been historically problematic because it is naturally above the ionization threshold 
of many types of targets and it happens on a time scale that favors the development of a large pre-plasma. There are widespread and obvious methods to keep the ASE level under control on the time scale of many tens of nanoseconds and above. For instance, the ASE can be temporally filtered out by use of fast optical switches like Pockels cells. Unfortunately, the laser pulse is temporally stretched during the amplification to a few nanoseconds so that the minimal temporal gate that can be applied equals the stretched pulse duration. And temporal gating after amplification turns out to be impractical because of the limitations imposed by the large beam dimension and fluence. As a result, light pulses amplified by CPA exhibit a nanosecond-long pedestal of constant power. The power of the nanosecond ASE pedestal is related to the noise power of the amplifier, i.e., the nanosecond temporal contrast $C_{n}$ of the laser is related to the signal-to-noise ratio of the amplifier:

$$
C_{n}=\frac{\text { Noise Power }}{\text { Laser Power }}
$$

The noise in CPA laser amplifier systems originates mostly from the first stage of amplification because the spontaneous emission photons getting amplified early on overwhelm the subsequently emitted spontaneous photons. In the case when the noise power is spatially limited to the laser mode itself, like in the case of amplification by regenerative amplification, only one spatial noise mode contributes to the ASE. For other types of laser pre-amplifiers based on multi-pass architectures, the many passes through the amplification medium acts like a soft aperture spatial filtering and this argument holds true. In high-gain single-pass pre-amplifiers like optical parametric amplifiers (OPAs), where self mode cleaning with diffraction or spatial filters cannot play an important role, the noise is not spatially limited to the laser mode and one has to differentiate between intensity and power contrast ${ }^{[5]}$. In the following calculation, the intensity contrast and power contrast are assumed equal (ASE limited to the laser spatial mode) and the ASE level is estimated for an amplifier whose noise originates in a regenerative amplifier, like at the PHELIX facility.

With the previous assumption, the determination of the temporal contrast restricts itself to the problem of the temporal noise in a one-dimensional quantum amplifier, where the noise in the high-gain approximation $(G \gg 1)$ equals to $1 / 2$ quantum per temporal mode that is degraded by a factor of two during amplification ${ }^{[6]}$. In addition, noise in a regenerative amplifier is emitted in two directions so a factor of two has to be added to the previous number. The power or intensity contrast ratio needs to take the laser peak power and noise power into account. The noise power is given by the product of photon energy by the gain of the amplifier, divided by the temporal extension of this photon, i.e., its coherence length. One can show that the duration of a noise temporal mode is then inversely proportional to the bandwidth of the amplifier:

$$
C_{n}=\frac{2 E_{\text {photon }} \times \frac{\text { Bandwidth }}{0.441} \times G}{P_{L}},
$$

where bandwidth/0.441 is the temporal duration of a noise mode (for a Gaussian pulse profile), $G$ is the gain factor of the laser amplifier, $P_{L}$ is the laser peak power and the factor 2 is specific to regenerative amplification. When the main pulse is compressed to the Fourier transform limit, the power of the pulse at the maximum divided by the gain and bandwidth gives the initial effectively seeded energy:

$$
C_{n}=\frac{2 E_{\mathrm{photon}}}{E_{\mathrm{in}}^{\mathrm{eff}}}=\frac{2}{N_{\text {Seed photons }}^{\text {eff }}}
$$

As a result, the ASE to laser peak power ratio or nanosecond temporal contrast $C_{n}$ only depends on the number of seed photons effectively participating to the amplification. This result is remarkably simple, it is also in good agreement with photometric estimates as proposed by Yariv $^{[7]}$, in which the equivalent input noise power for laser amplifiers $P_{\text {ASE, input }}$ is given (Equation (21.1-13), p. 570) in the approximation of a four-level laser system:

$$
P_{\mathrm{ASE}, \text { input }}=h v \mathrm{~d} v \frac{G-1}{G} .
$$

In the case of the regenerative amplifier with a gain much larger than unity, Equation (4) can be used to derive the ASE contrast, provided it is adapted to take into account the emission in two directions and one finds:

$$
C_{n}=\frac{2 h v \mathrm{~d} v}{P_{\text {seed }}^{\text {eff }}},
$$

where $h v$ is the photon energy, $\mathrm{d} v$ is the spectral bandwidth of the amplifier (supposed constant) and $P_{\text {seed }}^{\text {eff }}$ the effective seeded power into the amplifier, which can be written as:

$$
C_{n}=\frac{2}{N_{\text {seed photons }}^{\text {eff }}} \mathrm{d} v \mathrm{~d} t
$$

with $\mathrm{d} v \mathrm{~d} t$ the time bandwidth product of the amplifier that is assumed equal to one in this approximation (square pulse in time and frequency). It must be noted that the approximation of a constant spectral bandwidth for the photometric approach yields an error between 1 and 2 in the estimate of the pulse contrast ratio compared to the quantum noise approach that supposes Gaussian spectral intensity distributions.

Equation (6) is inversely proportional to the number of injected photons. However when the seed is very powerful, the contrast is not automatically very small as the own temporal contrast of the seed pulse can play a role and even become 
Table 1. Contrast as a function of the effective seed pulse energy (for a regenerative amplifier).

\begin{tabular}{lccccc}
\hline Seed & & & & & \\
energy & $1 \mathrm{pJ}$ & $100 \mathrm{pJ}$ & $10 \mathrm{~nJ}$ & $1 \mu \mathrm{J}$ & $100 \mu \mathrm{J}$ \\
$C_{n}$ & $3.8 \times 10^{-7}$ & $3.8 \times 10^{-9}$ & $3.8 \times 10^{-11}$ & $3.8 \times 10^{-13}$ & $3.8 \times 10^{-15}$ \\
\hline
\end{tabular}

dominant over the regenerative amplifier contribution. In the case when the seed pulse contrast is not infinite, this term must be added to Equation (6) as explained in Ref. [8]. As it is going to be seen, this argument does not hold for PHELIX as there the seed pulses are prepared using techniques that ensure a very high temporal purity.

Table 1 gives the nanosecond contrast ratio corresponding to a given effective seed energy for photons at $1053 \mathrm{~nm}$ using Equation (3). The first two columns cover the energy that is available from standard short-pulse laser oscillators. Such oscillators produce pulses in the nanojoule range that go first through the pulse stretcher whose transmission efficiency is generally much less than $50 \%$, especially when additional losses coming from the optical path and active elements like Pockels cell pulse pickers must be taken into account. The effective number of seed photons depends additionally on the spectral and spatial (mode matching) coupling efficiencies. In the case of a regenerative amplifier, it might take many round trips for the spatial mode to establish, inducing strong losses on the seed, while the noise photons fill the cavity mode automatically.

As a consequence, standard CPA laser systems exhibit contrast ratios around $10^{-6}-10^{-8}$ that are unfortunately not sufficient to avoid ionization and pre-plasma expansion in most of the target materials used in high-intensity lasermatter interaction experiments.

To avoid pre-plasma expansion, the intensity of the ASE pedestal must remain below a certain intensity threshold that depends on the material properties. This threshold is independent of the maximum intensity reached by the laser, so as a consequence, the signal-to-noise requirement increases with the peak power of the laser. For petawatt-level lasers capable of reaching intensities close to $10^{21} \mathrm{~W} \mathrm{~cm}^{-2}$, the nanosecond contrast requirement reaches up to $10^{-12}$ for the most sensitive targets. More generally, one can infer the ionization threshold of gases, dielectric and metals from data found in the literature on multi-photon ionization studies from gases and laser-induced damage threshold studies for solid targets. Because the ionization of gases is mostly intensity driven and collective effects can be neglected, this ionization threshold is time independent on the subnanosecond scale. For dielectrics, a damage threshold following a square root law is reported and commonly accepted ${ }^{[9]}$ at a level that is enhanced by the very small spot of the laser such that the band-gap collapse seen in largespot damage tests can be reduced ${ }^{[10]}$. For metals, a linear intensity dependency (constant fluence) is reported ${ }^{[11]}$ for

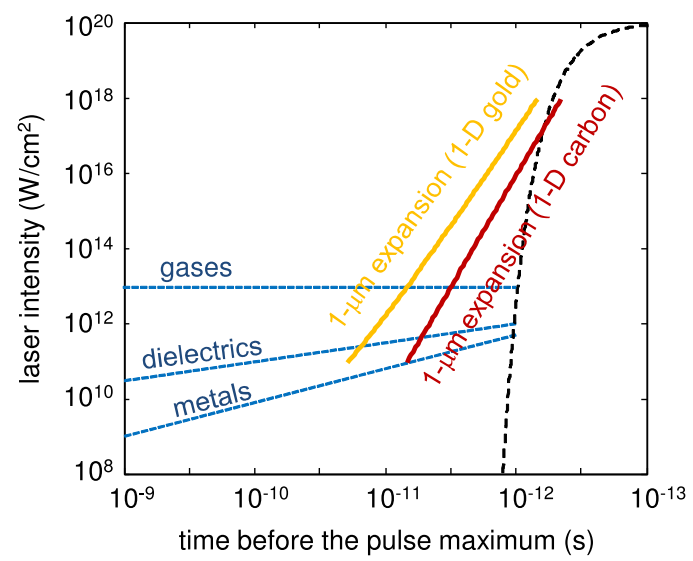

Figure 1. Intensity limits for ionization and the one-dimensional plasma expansion model from Samir et al. The dashed back line indicates a Gaussian pulse.

time scales from $10 \mathrm{ps}$ to $1 \mathrm{~ns}$. Figure 1, that summarizes the contrast requirements in view of pre-plasma generation, shows the typical ionization threshold intensities for gases, dielectric and metal in the sub-nanosecond regime as a function of the onset time of this intensity before the maximum of the pulse.

As a conclusion, the seed energy of a high temporal contrast amplifier must be boosted from the nanojoule to the many-microjoule range in order to fulfil the temporal contrast requirements, which assumes boosting the oscillator pulse energy by at least 4 orders of magnitude.

\section{Requirements on the picosecond rise time}

If the target remains unchanged thanks to a low-ASE level, the ionization threshold is reached during the rising slope of the pulse, and the target starts to expand into the vacuum. For sub-picosecond pulse lasers like PHELIX, this threshold might be reached several tens of picosecond before the maximum of the pulse and hydrodynamic effects play then a important role in the evolution of the target surface. Since the expansion time scale is at least an order of magnitude shorter, the resulting pre-plasma is however expected to be smaller than the pre-plasmas generated by ASE.

There is an analytical one-dimensional model describing this phenomenon, which can be used to get a rough estimate of the amount of plasma expansion for a given laser intensity. For this, the plasma is considered as isothermal, charge neutral and its electrons have a Maxwellian energy distribution $^{[12]}$. At $t=0$ equivalent to the time at which the threshold is reached, the plasma starts to expand into the vacuum and after an instant $\Delta t$ corresponding to the time when the peak intensity is reached, the density at the interface follows an exponential decay profile around a volume that is limited by the distance covered by the ionic sound wave. The solution is self-similar, that is, 


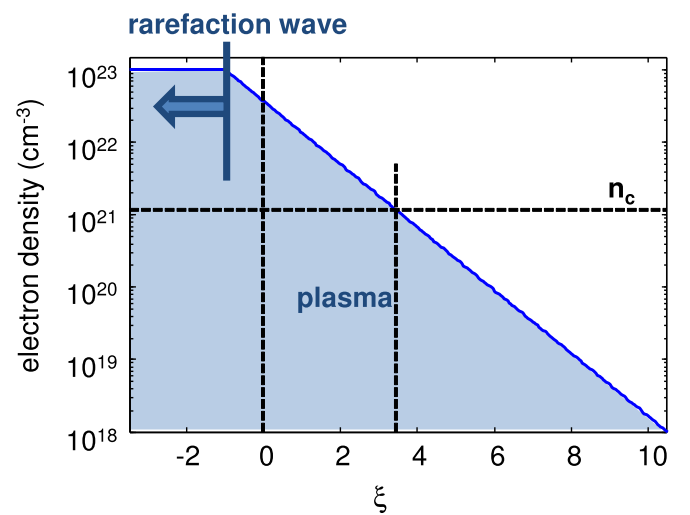

Figure 2. Self-similar analytical solution to the one-dimensional plasma expansion. $n_{c}$ : critical density

its shape does not change in time, and can be plotted for the dimensionless spatio-temporal parameter $\xi$ with $\xi=$ $x /\left(S_{0} \Delta t\right)$ where $\Delta t$ is the expansion time, $x$ the space coordinate and $S_{0}$ the ionic sound velocity:

$$
S_{0}=\sqrt{\frac{Z_{i} k_{B} T_{e}}{m_{i}}}
$$

where $Z_{i}$ is the ionization grade of the plasma, $T_{e}$ is the plasma electronic temperature and $m_{i}$ is the mass of the ions. In such a case, the plasma electronic density is given by:

$$
n(\xi)=n_{0} Z_{i} \mathrm{e}^{-(\xi+1)}, \quad \text { for } \xi>-1,
$$

where $n_{0}$ is the initial plasma ionic density. This simple model illustrated for a fully ionized hydrogen plasma at solid-state density in Figure 2 is valid for the first instants after the plasma has been created. It supposes the plasma temperature remains constant during the expansion and that radiative cooling is negligible. The one-dimensional aspect is also maintained as long as the plasma expansion remains small compared to the focal spot diameter.

Equation (8) is of interest because it links the position of a given plasma density to the time and electron temperature. In particular, one can find an expression of the location of the critical density of the plasma $x_{n c}$, that is the electron density at which the laser field is not able to propagate in the plasma cloud any more:

$$
\begin{aligned}
x_{n_{c}} & =S_{0}\left(\log \left(\frac{n_{0} Z_{i}}{n_{c}}\right)-1\right) \Delta t \\
& =\sqrt{\frac{Z_{i} k_{B} T_{e}}{m_{i}}}\left(\log \left(\frac{n_{0} Z_{i}}{n_{c}}\right)-1\right) \Delta t .
\end{aligned}
$$

As shown above, the position of the critical density depends on the electron temperature, which depends on the laser intensity. In other words, a higher laser intensity leading to a higher electron temperature and high plasma ionization grade will expand faster into the vacuum. For planar targets, a flow of the plasma over a distance of a laser wavelength $\lambda$ is in most cases tolerable and has little impact on propagation and the target areal density. This condition can be written as:

$$
\sqrt{\frac{Z_{i} k_{B} T_{e}}{m_{i}}}\left(\log \left(\frac{n_{0}}{n_{c}}\right)-1\right) \Delta t<\lambda
$$

When the plasma is hot and the charge-to-mass ratio large, the plasma expands faster while lower intensities can be tolerated for a longer time. To assess the criticality of this condition, the expansion time necessary for the critical density to reach $\lambda$ for various materials (gold and carbon) was calculated as a function of the laser intensity and compared to an ideal temporal profile in Figure 1. For the simulation, a relation between laser intensity and plasma temperature must be applied to Equation (10), which was assumed based on empirical plasma temperatures of 70 and $550 \mathrm{eV}$ at $10^{12}$ and $10^{15} \mathrm{~W} \mathrm{~cm}^{-2}$, respectively, as found in Refs. [13,14], with a scaling law following a power of $1 / 3$ of the intensity. This scaling is valid as long as the plasma is not relativistic, that is below $10^{18} \mathrm{~W} \mathrm{~cm}^{-2}$. Above this value, the light pressure cannot be neglected any more and the plasma does not expand in the same way as long as the laser pulse is 'on'. For comparison, a maximum laser intensity equal to $10^{20} \mathrm{~W} \mathrm{~cm}^{-2}$ and a Gaussian 400 -fs-long laser pulse is shown. The curve for carbon assumes a fully ionized carbon plasma $\left(C^{6+}\right)$. The gold plasma on the other hand is a bit more complicated to simulate because gold is not fully ionized at the lower intensities and the mean ionization grade depends on the laser intensity. The mean ionization grade for gold is then estimated by assuming that the ionization energy equals the plasma temperature (local thermodynamic equilibrium conditions). The ionization energies of the gold ions have been retrieved from the NIST database ${ }^{[15]}$. In addition, the time-dependent ionization threshold for metals and dielectrics are indicated in Figure 1 together with the ionization threshold for low density gases.

Altogether Figure 1 summarizes the nanosecond and picosecond contrast requirements for a laser pulse when an interaction with a mostly undeveloped plasma at the maximum of the laser intensity is desired. The important concepts of ionization threshold and minimum plasma hydrodynamic expansion are quantified showing that a slow rise of the pulse intensity on a time scale above ten picoseconds can yield a significant hydrodynamic flow of the plasma and changes in the interaction for the most sensitive targets.

\section{ASE tuning by means of uOPA}

The technique of ASE reduction by uOPA was first proposed and demonstrated by Dorrer et al. ${ }^{[16]}$ as a particularly 


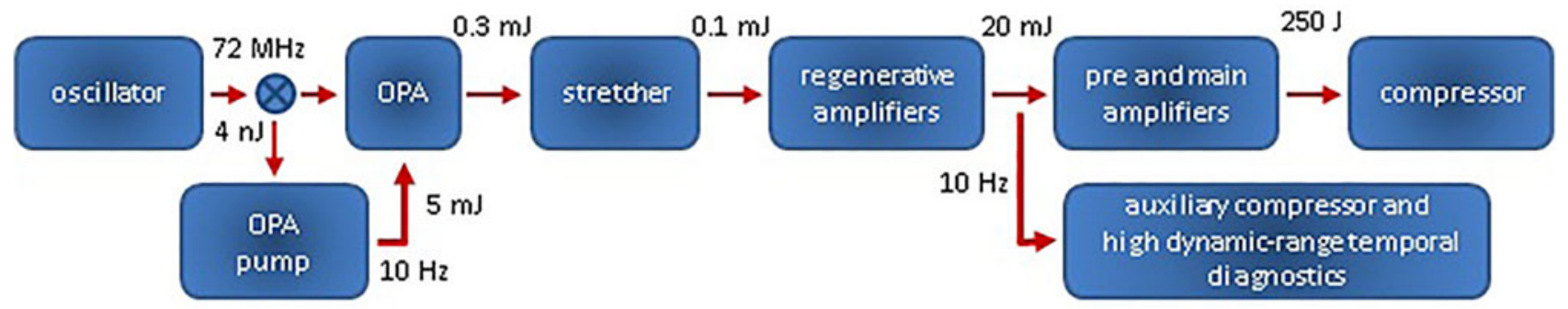

Figure 3. Schematics of the low-ASE version of the PHELIX laser commissioned in 2012. The auxiliary compressor is used for temporal contrast characterization on a daily basis.

practical solution for sub-picosecond lasers like the OmegaEP or PHELIX facilities. The schematics of the uOPA implementation at $\mathrm{GSI}^{[4]}$ is depicted in Figure 3, where the oscillator is used to seed a compact CPA laser (OPA pump) used as a pump for a synchronized sub-picosecond OPA. The details on the OPA pump laser are described elsewhere ${ }^{[17]}$. The rest of the chirped pulse amplifier is made of the short-pulse front end, pre and main amplifiers of the PHELIX facility ${ }^{[1]}$, whose front end comprises two sequential regenerative amplifiers. In addition to the uOPA stage, a series of 6 Pockels cells used as pulse pickers along the beam path between the stretcher output and the preamplifier output keep the nanosecond pre-pulses below the ionization threshold of the most demanding targets.

The pulse profile is measured using a scanning highdynamic-range cross-correlator (Sequoia, Amplitude Technologies SA). Because of the scanning nature of the measurement, only pulses amplified at high repetition rates can be measured. For this reason, an auxiliary compressor enables measuring the temporal profile of the pulses at the output of the regenerative amplifiers. We believe that this measurement is representative of the main fully amplified pulse of the laser facility because one of the features of large neodymium-doped glass lasers is the operation of the amplifiers far from saturation such that the main pulse and any noise features present at the regenerative amplifier output are similarly amplified with very little distortion. In addition, the fluence in the amplifiers stay below $1 \mathrm{~J} \mathrm{~cm}^{-2}$ such that the nonlinear effects and their influence on the temporal profile can be perfectly neglected.

For maximum temporal contrast operation, the full OPA pump pulse is used to drive the OPA into saturation to reach an energy of $300 \mu \mathrm{J}$, which represents a gain factor of about $10^{5}$. At this operation point, the energy fluctuations are also minimized and the stability of the nonlinear amplifier greatly improved. The jitter between the OPA pump and seed pulses is very low and not measurable with the accuracy of the setup (jitter $\ll 1$ ps peak to valley). Long term drifts are however present and could be attributed to temperature drifts happening during warm-up, as a Pockels cell driver used in the setup is getting warm. To counteract this effect, the OPA pump laser is usually left in operation $24 \mathrm{~h}$ per day,

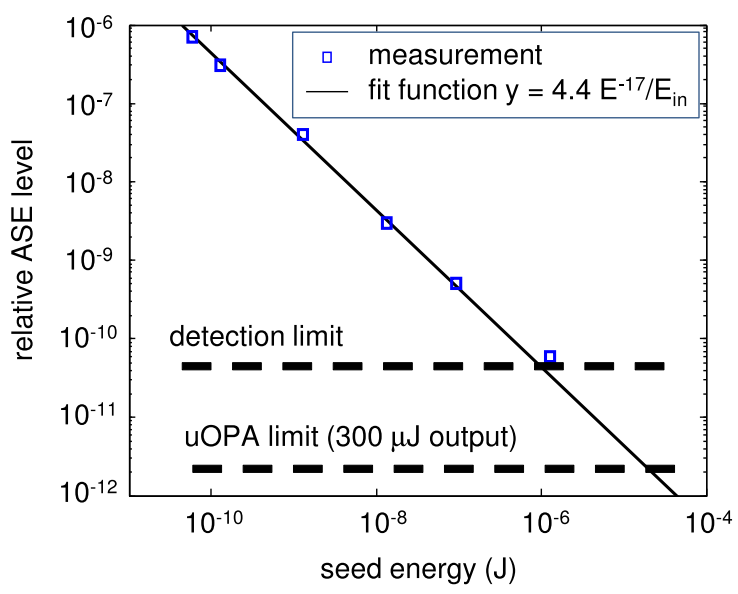

Figure 4. ASE level at PHELIX as a function of the seed energy measured at the regenerative amplifier stage input.

7 day a week during beam time. A steady state is usually reached within hours and a closed-loop routine locked on the pulse maximum energy is adjusting the time delay between pump and seed to offer hands-free stable operation over days without operator active adjustment.

While experiments aiming at generating an interaction between short pulses and ultra thin targets require the highest temporal contrast, other experiments may benefit from a light pre-plasma generation to improve the absorption of the laser pulse into the target. In such a case, the OPA gain is reduced and can be tuned continuously between $10^{5}$ and 1 . To compensate for the OPA gain, the amplification duration in the first regenerative amplifier is adjusted accordingly. Figure 4 shows the ASE level (dots) measured by a commercial scanning third-order cross-correlator at the end of the front end as a function of the seed energy. The seed energy is determined by taking into account the transmission through the stretcher (26\%), pulse picker $(80 \%)$ and injection Brewster surface $(25 \%)$, which amounts to $5 \%$ altogether. The Sequoia has a noise floor slightly below $10^{-10}$ and does not allow measuring the ASE level for the full OPA output energy. We could however verify that the ASE level scales linearly with the seed energy following the linear fit indicated in the plot (based of the least square method). 


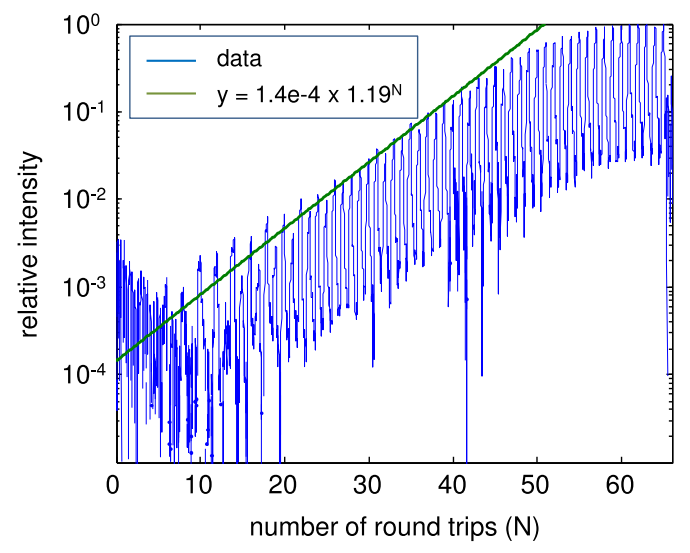

Figure 5. Measurement of the relative pulse energy as a function of the number of round trips in the regenerative amplifier. The part when $N \in$ $[20 \ldots 40]$ is used for the fit.

Note that the seed energy is different from the effective seed energy as given in Table 1 because our estimate does not take into account the coupling efficiency into the regenerative amplifier. To characterize this effect and verify if the data corroborate equation (3), we conducted an analysis of the regenerative amplifier build-up trace as shown in Figure 5. There, the blue curve shows an experimental measurement of the pulse energy in the regenerative amplifier as a function of the number of round trips. The regenerative amplifier is the first stage of amplification and it is separated from the OPA stage by the pulse stretcher and a pulse picker. As in the general case of regenerative amplification, the pulse starts between amplified in the small signal gain regime and reaches saturation after about 45 round trips as indicated in the plot shown with a logarithmic scale. For the first round trips, the pulse cannot be measured because of the electromagnetic noise picked by the photodiode and cable. In addition, the pulse energy does not grow smoothly but rather shows oscillations that indicate a poor mode coupling. When a fit is made on the part of the curve when the mode is established after 20 round trips but before saturation, the effective seed energy can be retrieved and equals $1.4(+0.6 /-0.2)$ $\times 10^{-4}$ times the regenerative amplifier output pulse energy. The output energy of the PHELIX regenerative amplifier equals $5 \mathrm{~mJ}$ so that the effective seed energy can be estimated to be 0.6 to $1 \mu \mathrm{J}$, which equals $3 \times 10^{12}-5 \times 10^{12}$ seed photons. From this measurement, the expected contrast ratio can be estimated by using Equation (3) and amounts to $4 \times 10^{-13}-6 \times 10^{-13}$ which falls within a factor of two of the estimate given on Figure 4. An explanation to this difference is that the ASE might be amplified on more than one spatial mode that results in a decrease of the temporal contrast ratio.

The effective seed energy is rather low compared to the OPA output energy but this difference can be explained by the losses happening between the OPA and regenerative amplifier (the transmission equals 5\%), while the remaining

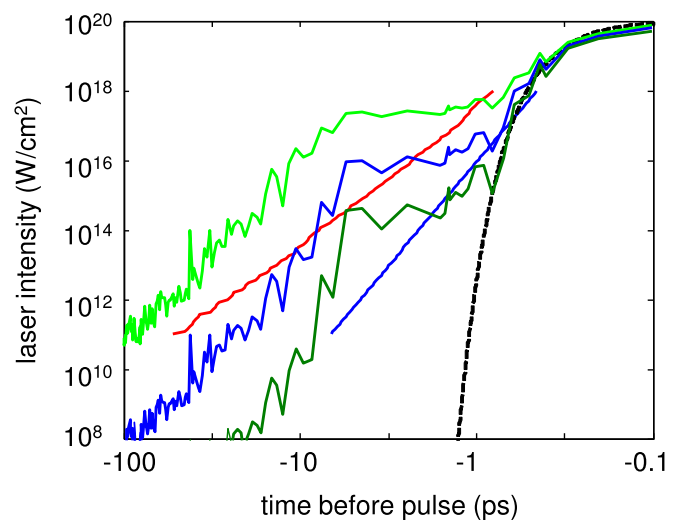

Figure 6. Comparison of the single (blue) and double (dark green) plasma mirror effect to the plasma expansion threshold for gold (red) and hydrogen (blue).

losses can be attributed to the coupling efficiency into the regenerative amplifier (5\%) (spatial and spectral).

\section{Pulse stiffening by means of plasma mirrors}

The nanosecond contrast at PHELIX has been estimated around $10^{-12}$ from gain measurement extrapolations (see Figure 4) and its positive effect on metallic targets has been experimentally reported in details elsewhere ${ }^{[18]}$, showing that the nanosecond contrast fulfils the ionization threshold requirement of even the most sensitive targets. However, the pulse profile at PHELIX (see Ref. [4] for instance) exhibits a slow rise time from the ASE background to a few orders of magnitude below the laser peak intensity, over about $100 \mathrm{ps}$. The origin of the slow rise time is still not known with certainty although studies in other high-energy CPA lasers systems might indicate that this temporal semi-coherent noise originates from the stretcher ${ }^{[19]}$. Figure 6 compares the temporal profile of the PHELIX pulse measured with a thirdorder cross-correlator with the predictions we derived from the model of Samir et al. The light green curve is the direct pulse measurement while the red and blue lines indicate the limits for a shift of the critical density of one micrometer for gold and hydrogen, respectively. From this, one clearly sees that the slow rise of the temporal intensity at PHELIX is a factor of 10 to 10000 larger than the requirement that we could derive from the model of Samir et al., and that a significant impact of the target expansion cannot be excluded. A possible deleterious effect is the appearance of an uncontrolled three-dimensional plasma flow, greatly reducing, for instance, the effective target areal density, due to the flow of the plasma outside the interaction focus.

The current solution to improve the interaction conditions is to use plasma mirrors. A plasma mirror is an antireflected coated surface that is located at PHELIX usually at 15 to $45 \mathrm{~mm}$ from the target (depending on the focusing geometry) such that the peak intensity reaches about 
$10^{15} \mathrm{~W} \mathrm{~cm}^{-2}$. Before the pulse arrives, the anti-reflecting coating reflects about $0.1 \%$ of the light to the target. When the intensity reaches the ionization threshold of the dielectric coating at about $10^{12} \mathrm{~W} \mathrm{~cm}^{-2}$, the mirror starts reflecting and its reflectivity increases with the laser intensity until it reaches a plateau for $10^{15} \mathrm{~W} \mathrm{~cm}^{-2}$ and above. The typically reflectivity of a single plasma mirror at PHELIX exceeds $80 \%$ because of the favorably S-polarized incoming beam and it can reach nearly $100 \%$ in specific cases ${ }^{[20]}$. As a result, a single plasma mirror is able to improve the temporal contrast by three orders of magnitude for all intensities found three orders of magnitude below the pulse maximum. The reflectivity curve of plasma mirrors has been repeatedly characterized at PHELIX before several beam times over a 24 month period and with focusing optics of various $F$ numbers. An example of such a measurement is shown in Figure 7. All measurements delivered comparable data as far as the reflectivity curve and maximum reflectivity are concerned. The data is fitted with an error function in order to define an intensity transfer function used on the second plot to make predictions on the pulse profile when one (blue curve) or two (dark green curve) plasma mirrors are used. As shown by the plot from Figure 6, a single plasma mirror is able to improve the pulse profile such that it fulfils the requirement of the plasma expansion model for heavier materials like gold. However a double plasma mirror might be necessary for light elements like solid hydrogen targets, $\mathrm{CH}_{2}$ foils and the like.

In addition, the comparison with the model seem to indicate that not only is the slow rise problematic for light target materials but also the pulse intensity between -10 and $-1 \mathrm{ps}$ seem to exceed by most the requirement. This indicates that this feature might be dominating the pre-plasma expansion at PHELIX. Efforts are currently applied to characterize this feature with more accuracy and find ways to improve it via static and/or active spectral phase correction.

\section{Conclusion}

The ASE temporal contrast in CPA laser amplifiers is best described in terms of quantum noise and can be reduced to a simple expression given by number of effectively seeded photons. We verified experimentally that the effective seed energy is much smaller than the seed energy at PHELIX, although a particular care has been taken to image relay the beam from the uOPA to the regenerative amplifier and mode matching has been implemented. This result can be extrapolated to other laser facilities and can be regarded as quite general as other laser facilities report similar performances. This work can be used to draw strategies for the next generation of high-intensity lasers planned to reach peak powers above $10 \mathrm{PW}$. In particular, one could opt for either an upscaled version of the temporal contrast boosting module or the development of techniques to improve the

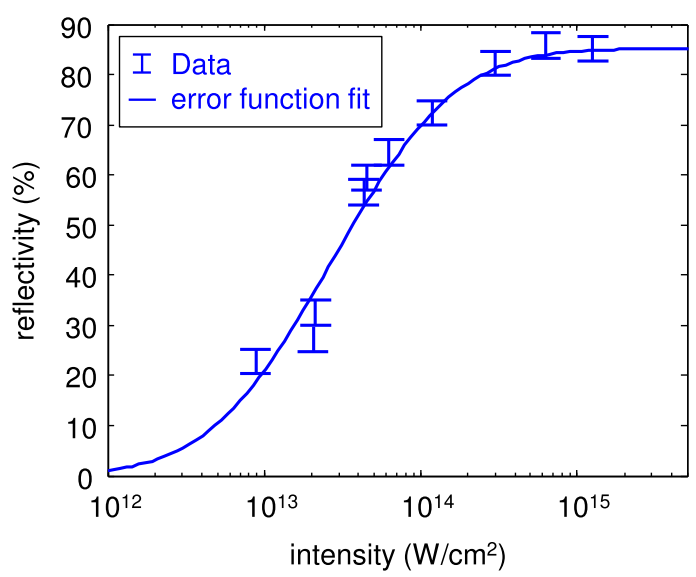

Figure 7. Plasma mirror reflectivity: data and fit based on an error function.

mode matching efficiency of the seed pulses into the chirped pulse amplifier.

For the leading edge of the pulse, our study show that pulses amplified at PHELIX probably induce some preplasma flow on a picosecond time scale and that measures to avoid it should be implemented when needed. For that, plasma mirrors represent a possible solution that do not reduce the on-target intensity significantly because of the high reflectivity of plasma mirrors used at $45^{\circ}$. However, this is only a palliative solution that should not hinder the search of temporally cleaner amplification schemes.

\section{References}

1. V. Bagnoud, B. Aurand, A. Blazevic, S. Borneis, C. Bruske, B. Ecker, U. Eisenbarth, J. Fils, A. Frank, E. Gaul, S. Goette, C. Haefner, T. Hahn, K. Harres, H.-M. Heuck, D. Hochhaus, D. H. H. Hoffmann, D. Javorková, H.-J. Kluge, T. Kuehl, S. Kunzer, M. Kreutz, T. Merz-Mantwill, P. Neumayer, E. Onkels, D. Reemts, O. Rosmej, M. Roth, T. Stoehlker, A. Tauschwitz, B. Zielbauer, D. Zimmer, and K. Witte, Appl. Phys. B 100, 137 (2010).

2. J. Itatani, J. Faure, M. Nantel, G. Mourou, and S. Watanabe, Opt. Commun. 148, 70 (1998).

3. M. P. Kalashnikov, E. Risse, H. Schönnagel, and W. Sandner, Opt. Lett. 30, 923 (2005).

4. F. Wagner, C. Joao, J. Fils, T. Gottschall, J. Hein, J. Körner, J. Limpert, M. Roth, T. Stöhlker, and V. Bagnoud, Appl. Phys. B 116, 429 (2014).

5. C. Dorrer, A. Consentino, and D. Irwin, Appl. Phys. B 122, 1 (2016).

6. H. Haus and J. Mullen, Phys. Rev. 128, 2407 (1962).

7. A. Yariv, Quantum Electronics (Wiley and Sons, New York, 1989), chap. 21.

8. X. Guo, X. Zou, Y. Xu, X. Lu, Y. Li, C. Wang, Y. Leng, and R. Li, Opt. Commun. 334, 323 (2015).

9. B. C. Stuart, M. D. Feit, A. M. Rubenchik, B. W. Shore, and M. D. Perry, Phys. Rev. Lett. 74, 2248 (1995).

10. C. Carr, H. B. Radousky, and S. G. Demos, Phys. Rev. Lett. 91, 127402 (2003). 
11. P. B. Corkum, F. Brunel, N. K. Sherman, and T. Srinivasan-Rao, Phys. Rev. Lett. 61, 2886 (1988).

12. U. Samir, K. Wright, and N. Stone, Rev. Geophys. 21, 1631 (1983).

13. R. Bleach and D. Nagel, J. Appl. Phys. 49, 3832 (1978).

14. F. Young, R. Whitlock, R. Decoste, B. Ripin, D. Nagel, J. Stamper, J. Mcmahon, and S. Bodner, Appl. Phys. Lett. 30, 45 (1977).

15. http://physics.nist.gov/PhysRefData/ASD/ionEnergy.html. The ionization energies for gold can be found from the NIST database - Accessed: 01/12/2015.
16. C. Dorrer, I. A. Begishev, A. Okishev, and J. D. Zuegel, Opt. Lett. 32, 2143 (2007).

17. C. P. João, F. Wagner, J. Körner, J. Hein, T. Gottschall, J. Limpert, and V. Bagnoud, Appl. Phys. B 118, 401 (2015).

18. F. Wagner, S. Bedacht, A. Ortner, M. Roth, A. Tauschwitz, B. Zielbauer, and V. Bagnoud, Optics Express 22, 29505 (2014).

19. C. Hooker, Y. Tang, O. Chekhlov, J. Collier, E. Divall, K. Ertel, S. Hawkes, B. Parry, and P. Rajeev, Opt. Express 19, 2193 (2011).

20. G. Scott, V. Bagnoud, C. Brabetz, R. Clarke, J. Green, R. Heathcote, H. Powell, B. Zielbauer, T. Arber, P. McKenna, and D. Neely, New J. Phys. 17, 033027 (2015). 